\title{
AMGA: An Adaptive and Modular Genetic Algorithm for the Traveling Salesman Problem
}

\author{
Ryoma Ohira, Md. Saiful Islam, Jun Jo, Bela Stantic \\ Griffith University, Southport QLD 4215, Australia \\ $\{r$.ohira, saiful.islam, j.jo, b.stantic\}@griffith.edu.au
}

\begin{abstract}
The choice in selection, crossover and mutation operators can significantly impact the performance of a genetic algorithm (GA). It is found that the optimal combination of these operators are dependent on the problem characteristics and the size of the problem space. However, existing works disregard the above and focus only on introducing adaptiveness in one operator while having other operators static. With adaptive operator selection (AOS), this paper presents a novel framework for an adaptive and modular genetic algorithm (AMGA) to discover the optimal combination of the operators in each stage of the GA's life in order to avoid premature convergence. In AMGA, the selection operator changes in an online manner to adapt the selective pressure, while the best performing crossover and mutation operators are inherited by the offspring of each generation. Experimental results demonstrate that our AMGA framework is able to find the optimal combinations of the GA operators for each generation for different instances of the traveling salesman problem (TSP) and outperforms the existing AOS models.
\end{abstract}

Keywords: Adaptive Genetic Algorithm · Modular Genetic Algorithm · Adaptive Operator Selection · Traveling Salesman Problem

\section{Introduction}

The traveling salesman problem (TSP) is a combinatorial optimization problem that is derived from many production and scheduling problems and is presented as a salesman being required to visit $n$ cities but given the traveling costs between each city, the salesman is to find the shortest distance required to visit all required cities [1]. When approaching the problem with a genetic algorithm (GA), the genotype represents the order in which a salesman visits the cities. As such, mutations and crossover must ensure that no city is visited more than once. While this places restrictions on the techniques used for the selection, crossover and mutation approaches, studies identify each step with having multiple techniques and methods that aim to improve a GA's output.

\subsection{Related Work}

A comprehensive study into the crossover operators and mutation operators demonstrate that the choice in operators can directly impact a GA's performance 
[2]. This was supported by studies into the effects of contemporary mutation [3] and crossover [4] operators for the TSP. Comparative studies of selection operators also demonstrate that the choice in selection operator has an effect on both the convergence [5] and time complexity [6]. By analyzing the results from these comparative studies, it can be seen that improving the effectiveness of a GA for the TSP is a multi-dimensional problem resulting from the combination of selection, crossover and mutation operators along with the problem size.

Existing literature $[7,8]$ identify a number of trends emerging in exploring adaptive operators for GAs, specifically operator selection. There are a number of works for introducing adaptiveness to GAs through AOS. Spears [9] introduces adaptiveness into the crossover phase with the 1Bit Adaptive Crossover (1-BX) where the selected crossover operator is encoded into the genotype. While limited to two operators, Spears identified that by simply making more operators available, the GA experienced significant improvements in performance. Riff and Bonnaire [10] extended this concept to include more operators. Gomez proposed the HAEA hybrid adaptive GA [11] which encodes the operator rates and rewards operators according to whether an offspring is better or worse than its parents. Cruz-Salinas and Perdomo [12] extend on this work by having population of operators that are exposed to evolution and mutation while maintaining the operator selection method employed by HAEA. However, the AOEA algorithm employs operators that are not suitable for the ordered nature of the TSP. Montero and Riff $[13,14]$ propose self-adaptive operator selection by including the probabilities of the operators in the gene. Two methods are proposed: a random reward/penalty value and a ratio on the difference between an offspring and parents' fitness in comparison to results from the last generation. Montero and Riff also suggested a global operator probability with a single operator selected for the next generation.

The AMCPA proposed by Osaba et al. $[15,16]$ is another implementation of the AOS where a new crossover operator is selected when it is beneficial to the search process. The crossover probability adapts according to the search performance on recent generations and the current generation number. This enables the algorithm to prevent premature convergence and also allows the AMCPA to use multiple crossover operators which are applied alternatively. The AMCPA only applies AOS for the crossover operator and makes the assumption that the 2-opt mutation operator is the most suitable for all instances of the TSP. Furthermore, the AMCPA was only compared to the GA for TSP instances where $n \leq 500$. While Spears demonstrated that the availability of multiple crossover operators improves performance, Osaba et al demonstrate that self-adapting operator selection can enable a GA to further explore the problem space.

\subsection{Contribution}

Unlike the existing works, AMGA proposed in this paper employs self-adapting operator selection to each stage of the GAs process. AMGA is randomly assigned a selection operator when initiated. The probability of the selection operator changing is calculated according to the number of generations where there are no 
Table 1: Comparison of Self-Adaptive Operator Selection GAs

\begin{tabular}{c|l}
\hline Genetic Algorithm & Adaptive Operator Selection (AOS) \\
\hline \hline Static GA & $\begin{array}{l}\text { No AOS } \\
\text { One of two crossover operators are encoded into the genotype } \\
\text { and inherited by child. } \\
\text { Inheritance of multiple crossover operators. } \\
\text { AOS crossover operators with rewards and penalties for improve- } \\
\text { HAEA [11] } \\
\text { ments in offspring. } \\
\text { Random selection between multiple crossover operators once } \\
\text { population health declines. } \\
\text { Crossover operators are presented as trees and are subject to } \\
\text { evolution. Probability of selecting an operator is dependent on } \\
\text { its performance. } \\
\text { Selection between multiple selection, crossover and mutation op- } \\
\text { erators. }\end{array}$ \\
\hline
\end{tabular}

significant improvements in fitness. This ensures that the algorithm responds to selective pressure in an on-line manner. Once a selection operator is chosen, it is used to select the parents for creating a new generation of offspring. The crossover and mutation operators for each individual is encoded into the genotype as extra genes. Once two parent individuals are selected, the crossover and mutation operators of the fittest parent is employed and inherited by the offspring.

Although crossover operators have been encoded into the genotype in previous works $[9,10]$, the AMGA includes both crossover and mutation operator genes. The crossover and mutation operators made available to the GA aims to recover the diversity lost by the selection stage while maintaining a high level of overall fitness in the population. Where existing literature has focused on the performance gains made by introducing AOS to the crossover stage, results from experiments demonstrate that by making more operators available for each stage of the process, AMGA is able to maintain diversity for more generations and avoid premature convergence.

Table 1 compares the self-adaptive operator selection in AMGA with the existing works in the literature.

\subsection{Organization}

The paper is organized as follows: Section 2 reviews the selection, crossover and mutation operators experimented in this paper; Section 3 presents our framework of adaptive and modular genetic algorithm for solving travelling salesman problem; Section 4 presents the experimental evaluation of our framework; finally Section 5 concludes the paper.

\section{Background}

\subsection{Selection Operators}

The selection operator phase in the GA is responsible for determining which individuals are used for the crossover phase. This paper looks at the effects of the most commonly used selection operators for GAs as discussed below. 


$$
\begin{aligned}
& \begin{array}{l|l|l|l|l|l|l|l|l|}
\cline { 2 - 7 } \text { Parent } 1 & 1 & 2 & \mathbf{3} & \mathbf{4} & \mathbf{5} & \mathbf{6} & \mathbf{7} & 8 \\
\hline
\end{array} \\
& \text { Child } \begin{array}{r|l|l|l|l|l|l|l|}
8 & 7 & 3 & 4 & 5 & 6 & 1 & 2 \\
\hline
\end{array} \\
& \begin{array}{ll|l|l|l|l|l|l|l|}
\multirow{2}{*}{\text { Parent } 2} & 8 & 4 & 6 & 7 & 5 & 1 & 3 & 2 \\
\cline { 2 - 8 } & & & & &
\end{array}
\end{aligned}
$$

Fig. 1: Partially Mapped Crossover

$$
\begin{aligned}
& \text { Parent 1 } \begin{array}{|l|l|l|l|l|l|l|l|}
\hline 1 & 2 & 3 & 4 & 5 & 6 & 7 & 8 \\
\hline
\end{array} \\
& \text { Child } \begin{array}{l|l|l|l|l|l|l|l|}
\hline & 2 & 5 & 4 & 6 & 3 & 7 & 8 \\
\hline
\end{array} \\
& \begin{array}{l|l|l|l|l|l|l|l|l|}
\cline { 2 - 7 } \text { Parent 2 } & \mathbf{2} & \mathbf{1} & \mathbf{4} & \mathbf{8} & 5 & 6 & 3 & 7 \\
\hline
\end{array}
\end{aligned}
$$

Fig. 3: Modified crossover operator

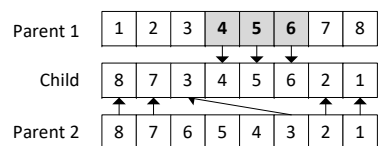

Fig. 2: Ordered Crossover Operator

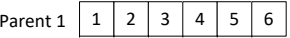

$$
\begin{aligned}
& \begin{array}{l|l|l|l|l|l|l|}
\cline { 2 - 6 } \text { Child } & 2 & 3 & 4 & 5 & 1 & 6 \\
\hline
\end{array} \\
& \begin{array}{l|l|l|l|l|l|l|}
\cline { 2 - 7 } \text { Parent } 2 & 2 & 4 & 3 & 1 & 5 & 6 \\
\hline
\end{array}
\end{aligned}
$$

Fig. 4: Edge recombination operator

Fitness Proportionate Selection (FPS) FPS is a commonly used operator for selecting parents for crossover where the probability of being selected, $p_{i}$, is proportional to the fitness in relation to the overall fitness of the population [17]. This is expressed in Eq. 1 where $f_{i}$ is the fitness of the given individual, $i$, from a population of size $n$. FPS can result in conditions where

$$
p_{i}=\frac{f_{i}}{\sum_{j=1}^{N} f_{j}}
$$
the GA lacks selective pressure in exploring the problem space or prematurely converge when the search space has narrowed down too quickly [18].

Rank Based Selection (RBS) RBS is proposed to eliminate the disadvantages of FPS [19]. This operator orders the individuals by their fitness where the worst performing individual is assigned a rank value of 1 and the best performing individual is assigned $n$. This can be expressed as in Eq. 2 where $i \in 1, \ldots, N$. $\mu^{+}$is the expected maximum value of the individual with rank $n$ where $\mu^{+} \geq 0$. $\mu^{-}$is the minimum expected value of the individual of rank 1 with the constraint $\mu^{+}=2-\mu^{-}$. Baker notes that a $\mu^{+}$with a

$$
p_{i}=\frac{1}{N}\left(\mu^{-}+\left(\mu^{+}-\mu-\right) \frac{i-1}{N-1}\right)
$$
value of 1.1 generates optimal results with it out performing fitness proportionate selection on the selected problems [19]. While ranking removes the problems associated with scaling and prevents the magnitude of the fitness differences impacting the selective pressure, it can increase computation time [6].

Tournament Selection (TNS) TNS randomly selects candidates from the population with the best one being selected for the next step. While tournaments are usually between two individuals, a generalized method can be written with a variable size. As a tournament selection can be executed with a single pass, time complexity is $\mathcal{O}(N)$ as it does not require any sorting [6].

\subsection{Crossover Operators}

Crossover operators are responsible for generating new combinations for genotypes by combining two parents to create offspring.

Partially-Mapped Crossover(PMX) PMX produces children by choosing a sub-sequence of genes from one parent while preserving the order and position of 


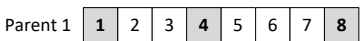

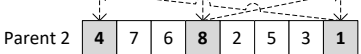

Fig. 5: Cycle Crossover: Defining the cycle

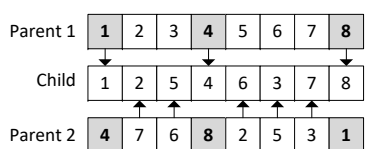

Fig. 6: Cycle Crossover Operator

the genes from another parent. An initial sequence of genes between points $i$ and $j$ are copied from one parent to the child. The genes between $i$ and $j$ from the second parent are mapped to new positions using the gene positions from parent 1 if they do not already exist in the child. The remainder genes are inherited from parent 2 if they do not exist in the child. This mapping and tracing process ensures the child's genes are subsequences inherited from both parents. In Fig. 1 , the child directly inherits the sequence of $3,4,5$ and 6 from parent 1 . The gene with the value 6 is in the same positions for parent 2 and can be ignored. Gene 7's position is taken by gene 4 and so is then mapped to the position of gene 4 from parent 2 . This can be seen again where the gene 1 is mapped to the position of gene 3 in parent 2 through: $1 \rightarrow 6 \rightarrow 3$. The remainder genes are then inherited directly from parent 2 .

Ordered Crossover Operator (OX) OX selects a sequence between points $i$ and $j$ of one parent and then inserts the genes from the second parent in the order they present themselves while ensuring that the child does not contain duplicate values [20]. The process can be seen in Fig. 2. The modified ordered crossover operator (MOX) in Fig. 3 selects a random point, $i$, within the order of both parents. The sequence to the left of the point for parent 2 identifies the genes from parent 1 that will maintain their position and order. The child inherits the remaining genes from parent 2 .

Edge Recombination Operator (ERO) ERO examines the link between each node and creates an edge map to construct offspring that inherit as much information as possible from the parent genes [21]. An initial node is selected from the edge list that has the largest number of edges. In Fig. 4, both nodes 1 and 2 have four edges and node 2 is randomly chosen. The node with the fewest edges are considered with one chosen for the next part. In the example, nodes 3,4 and 6 all have two edges and node 3 is chosen randomly. Node 3 has edges to nodes 1 and 4 , with 4 having the fewest edges and is thus chosen. This is continued until the child genotype is filled.

Cycle Crossover Operator(CX) In CX, a mapping cycle is initiated at a randomly selected point $i[1]$. In Fig. 5 , given that $i=4$, the cycle can be seen to be $4 \rightarrow 8 \rightarrow 1 \rightarrow 4$. The child then inherits these values and their positions from parent 1 with the remainder filled from parent 2 as seen in Fig. 6.

\subsection{Mutation Operators}

The purpose of a mutation operator is to maintain genetic diversity to prevent premature convergence. As with the selection and crossover operators, a number of mutation operators exist and contribute to a GA to varying degrees. 


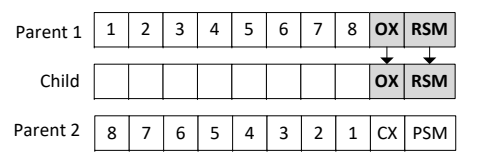

Fig. 7: AMGA operator encoding

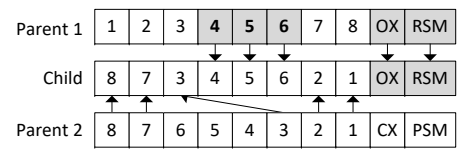

Fig. 8: AMGA crossover

Center Inverse Mutation (CIM) CIM divides the genotype into two sections from a randomly selected point. The order of genes in each subsection is reversed. This method results in a major variation to the original genotype [3].

Reverse Sequence Mutation (RSM) RSM selects a sequence between two randomly selected points $i$ and $j$ where $i<j$ [3]. The order of the genes within the sequence is then reversed. This ensures that the original order is partially preserved with a $p_{m}$ probability of mutation occurring.

Partial Shuffle Mutation (PSM) PSM swaps a gene given a probability of $p_{m}$ with another randomly selected gene [3]. This partially preserves the original order of genes but also allows noncontinuous mutation to occur.

\section{AMGA: An Adaptive and Modular Genetic Algorithm}

While studies into the performance of different methods for selection, crossover and mutation demonstrate how choices of an operator can directly affect the performance of a GA, the results suggest that an optimal combination for a given problem is dependent on the characteristics and size of the problem space. When considering operators as additional dimensions to an optimization problem, it becomes clear why this topic has been the subject of research since the 1980 's. This problem can be simplified as an optimization of the three operational phases.

- Selection operators immediately introduce selection pressure and time complexity to a GA. While tournament selection supports a GA in exploring the problem space, a rank or tournament selection operator produces more selection pressure in exploiting available solutions.

- Crossover operators introduce a range of solutions with different priorities between time complexity, order preservation and the bias in the makeup of new offspring. The goal of a crossover operator being the preservation of good genes while replacing the genes that are counter productive to its goals.

- Mutation operators being responsible for maintaining a healthy level of genetic diversity in the population to ensure that the GA does not converge prematurely. The importance of which can be seen when the diversity lost through selection and crossover isn't adequately replaced. The mutation operators themselves provide a varying degree of success in maintaining this genetic diversity but no substantial study has been done into the effects of the combination of the three operational phases.

In the proposed AMGA, each operational phase is implemented in a modular fashion where each operator is included as a module which can be selected 


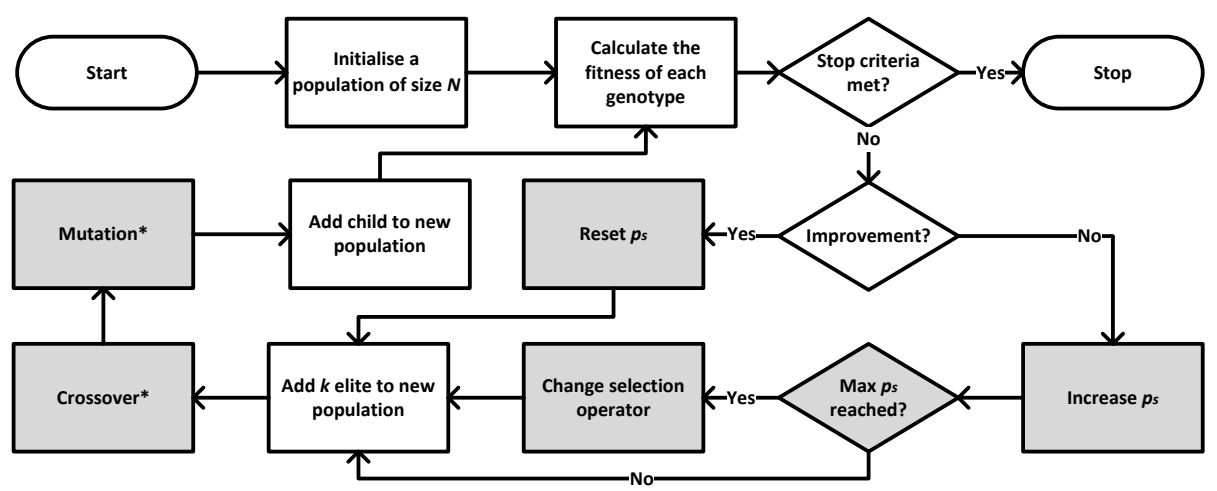

Fig. 9: Schematic diagram of our proposed AMGA framework

and changed with another if the algorithm reaches a local optima. In Fig. 9, the highlighted sections indicate the logic implemented for adaptive selection in operators. The selection operator is the first step where adaptive operator selection is implemented. This is done by implementing a new selection parameter, $p_{s}$. When a population is initialized, a selection operator is randomly selected and $p_{s}$ is given a value of 0 . As the population converges and no improvement is made to the fitness, the $p_{s}$ value is incremented until $p_{s} \geq 1$. This is expressed in Equation 3 where $g$ is the number of generations since the last improvement in fitness, $k$ is the current generation and $n$ is the problem size. As $g$ indicates a lack of progress, the AMGA is able to quickly respond to this by the way $g$ scales. The rate at which $p_{s}$ increases also scales with the size of the problem. Both of these features allow for the AMGA to explore with one selection operator for longer with larger problems. However, this still enables the AMGA to switch to another selection operator if the selected operator is inefficient or a change in selective pressure is needed. Furthermore, as the algorithm produces more generations, it becomes more agile in switching between operators.

The crossover and mutation operators are encoded into the genotype itself, as demonstrated in Fig. 7. The $p_{s}=p_{s}+\frac{2 g+k}{n^{2}}$ initial population is randomly assigned a crossover and mutation operator. During the crossover phase, the child genotype inherits the mutation and crossover operators of its fittest parents. The inherited crossover operator is then applied between the two parents to create the new offspring. This offspring is then subject to the inherited mutation operator with the probability of $p_{m}$. The key aspects of the AMGA can be identified as:

- Selection as an adaptive operator selected randomly but changed when the algorithm fails to experience improvement. Operators included as modules are the tournament, rank and fitness proportionate selection operators. The probability of the operator changing is defined as $p_{s}$ and is dependent on the size of the problem, the current generation and the number of generations since an improvement in fitness was recorded. 
- Crossover and Mutation are encoded into the genotype with offspring inheriting the operators of the fittest parent as demonstrated in Fig. 7. The inherited crossover and mutation operators are applied as in Fig. 8.

- Mutation probability affects crossover and mutation operators where these have a $p_{m}$ probability of changing to a new operator. This supports the goal of maintaining genetic diversity in both the genotype and the operators.

The operators for the AMGA were selected for their performance in existing literature and their known contribution to a GA's performance. For the insertion phase, an elitist strategy which involves selecting $k_{1}$ best performing genotypes is implemented. Furthermore, the $k_{2}$ worst performing genotypes are also included for increasing the minimal genetic diversity. The remaining population genotypes are created using the selection, crossover and selection strategies outlined above.

\section{Experiments}

This section demonstrates the experimental evaluation of our proposed framework of adaptive and modular genetic algorithm (AMGA) for optimizing travelling salesman problem.

Test Functions Tests from the Travelling Salesman Problem Library (TSPLIB) synchronous benchmark tests were selected for their variety in $n$ size and optimum distance. Equation 4 models the convergence criteria where the algorithm ends execution when the algorithm does not experience an improvement in its fitness for $g$ generations. With $n$ being the size of the problem and $k$ the current generation of the algorithm. This ensures that the convergence criteria scales to

$$
c=n+\sum_{k=1}^{n} k
$$

both parameters and scales in a way that allows for larger problems to explore longer before being classified as having reached a convergence point. Each TSPLIB test is run 50 times with average distance, standard deviation for distance and average processing being calculated. Furthermore, the two-sample z-tests on the

$$
z=\frac{\overline{x_{1}}-\overline{x_{2}}}{\sqrt{\frac{\sigma_{1}^{2}}{n_{1}}+\frac{\sigma_{2}^{2}}{n_{2}}}}
$$
mean distances between the GA and AMGA as well as the AMCPA and AMGA have been included. The two-sample z-test is chosen as it demonstrates the significance of the differences between two means and is particularly effective for cases where the sample size is greater than 30 . This is expressed as in equation 5 , where $x_{i}$ is the mean distance, $\sigma_{i}^{2}$ is the standard deviation and $n_{i}$ is the sample size of tests for algorithm $i$. A positive value indicates a significant improvement of $\overline{x_{1}}$ over $\overline{x_{2}}$ where as a negative value indicates a significant decrease in performance. Alternatively, a neutral value means that there is no significant improvement or decrease in performance. This ensures that an improvement in performance offered by the AMGA can be quantified.

Genetic Algorithm Operators For static genetic algorithms, the following crossover operators are incorporated: PMX, MOX, OX, CX, ERO. Mutation operators include: CIM, RSM, PSM. Selection operators are: Fitness Proportionate, Rank and Tournament. Each combination of selection, crossover and mutation 
Table 2: AMGA Components

\begin{tabular}{|c|c|}
\hline Component & Operators \\
\hline \hline Selection & FPS, RBS, TNS \\
Crossover & PMX, MOX, OX, CX, ERO \\
Mutation & CIM, RSM, PSM \\
\hline
\end{tabular}

operator is tested under the same conditions to ascertain the best performing combination. This is combination and its results are then used to represent static GAs for the given problem and acts as the control for this experiment.

The AMCPA was implemented as described by Osaba et al for the TSP. The parameters for $p_{c}$ and the operators available for the algorithm were also maintained. The AMCPA itself was implemented in the same language as the control and AMGA. The AMGA incorporated the operators outlined in Table 2. These components were chosen due to their contribution in improving the performance of the static GAs for optimum solutions and computation times.

Computing Environment The algorithms are executed on a Intel Core i73770 CPU with 32GB main memory. The software is written in Python 2.7 and executed using the PyPy interpreter with Numpy.

\subsection{Results}

As stated previously, the best performing combinations for each TSPLIB instance is presented as the control for the experiment and can be seen in Table 3. From the table, it can be seen that the optimal combination of crossover, selection and mutation operators varies with the size of the problem. While the smaller problems benefit from the OX crossover operator, the MOX has a greater contribution to the GA's performance for the larger problems. While the tournament selection operator is present in the majority of test instances, it should be noted that there was no statistically significant difference in mean distance between the tournament and rank selection operators. However, the GAs with the tournament selection would tend to converge in a shorter time period. This is likely due to the time complexity outlined in previous sections. The difference between the operator combinations for GAs become more significant as the problem size increases, this highlights the problem of determining the optimal operator combination for any given problem.

The AMCPA was originally tested against a GA with a different set of operators. While the AMCPA has improved performance over the static GAs, the magnitude of these improvements differ from the findings by Osaba et al. [15]. These results confirm the robustness of adaptive operator selection in a GA. In particular, the tests where $100<n<300$ show a significant increase in performance and efficiency. However, the diminishing improvements for the larger problems suggests a limit to the degree in which an adaptive crossover operator selection is able to contribute to the overall improvement of a GA. The AMCPA algorithm was chosen as the benchmark due to its proven performance in the TSP. This premature convergence suggests that diversity is not adequately maintained in the later generations for the larger problem spaces in AMCPA. 


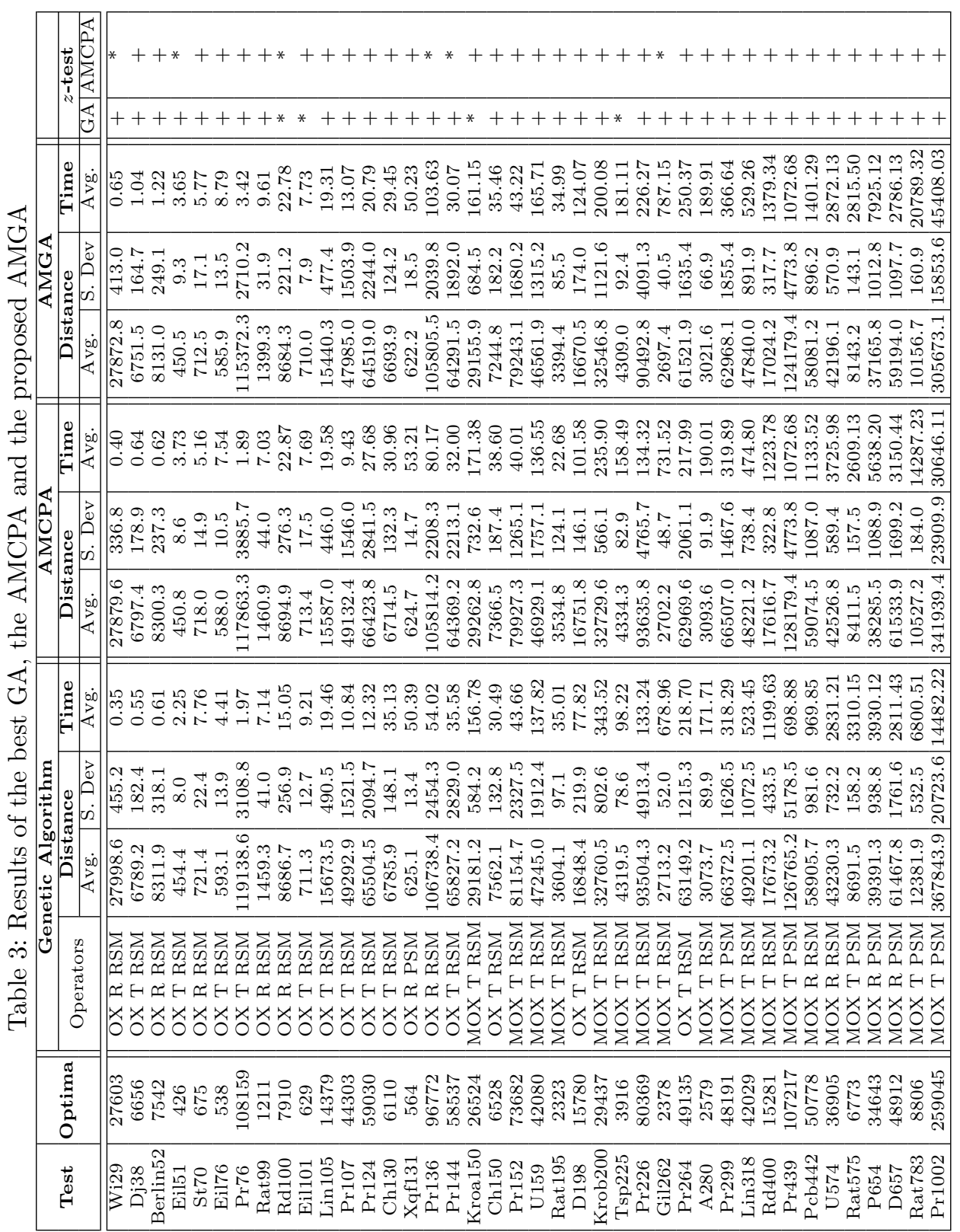


Performance Analysis Results by the AMGA show that introducing AOS in multiple phases increases the number of generations an algorithm is able to run before converging on a solution. That most instances showing lower standard deviation suggests that the AMGA is generally more consistent than the either the GA or the AMCPA. As the algorithms are run until there are no further improvements, the AMGA can be seen to have run for a longer time. This suggests that implementing adaptive operator selection to the mutation and selection phases enables the algorithm to maintain a higher degree of genetic diversity, particularly in the later generations. The results from the two-sample $z$-test in Table 3 indicate instances where the AMGA has a significant improvement over the GA or the AMCPA with a + . Results with a $*$ indicates no significant improvement. Of the 40 TSPLIB instances, the AMGA has statistically significant improvements in performance for 36 instances $(90.0 \%)$ against the static GAs and for 34 instances (85.0\%) against the AMCPA. Of these instances, there was only one instance (Rd100) where the AMGA did not demonstrate a significant improvement over either the GA or AMCPA. As the AMGA is converging at later generations, it can also be seen as having a greater mean time for computation. This is more pronounced as the problem size increases. Thus by introducing AOS to all phases of the process, the proposed AMGA is able to maintain a healthy level of population diversity for a longer period of time.

Convergence Analysis By examining the data collected from each algorithm across all the runs, a number of characteristics of the AMGA can be identified. An example of the AMGA converging later with larger problem sets is shown in Fig. 10. In Fig. 10a the AMCPA and GA converged between 2000 generations of one another where as the AMGA has produces another 4000 generations. This pattern can also be seen in Fig. 10b where the AMCPA and AMGA produce 5000 to 10000 more generations than the static GA. For problems with an even larger $n$ size, the AMGA exhibits greater performance gains. The results for test U574 shown in Fig. 10c demonstrate that for a medium size problem the AMCPA and AMGA run for a similar number of generations in comparison to the static GA. For a large problem where the $n$ size is 783 for Rat783, the AMGA can be seen as running for even longer than the AMCPA with both significantly more effective than the static GA as shown in Fig. 10d.

\subsection{Summary}

The above findings point to the adaptive qualities enabling the AMGA to maintain a healthy level of genetic diversity in its population for longer. This allows the algorithm to continue evolving for more generations before converging on a solution. Furthermore, the improvements in computation time suggests that the algorithm is using operators with lower time complexity for a majority of the time. As the algorithms meet the convergence criteria before reaching the known optimal solution, introducing greater control and monitoring techniques on the genetic diversity may result in further improvements. 


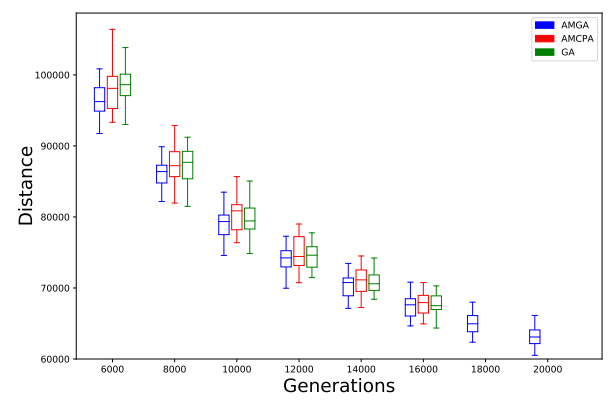

(a) $\operatorname{Pr} 299(n=299)$

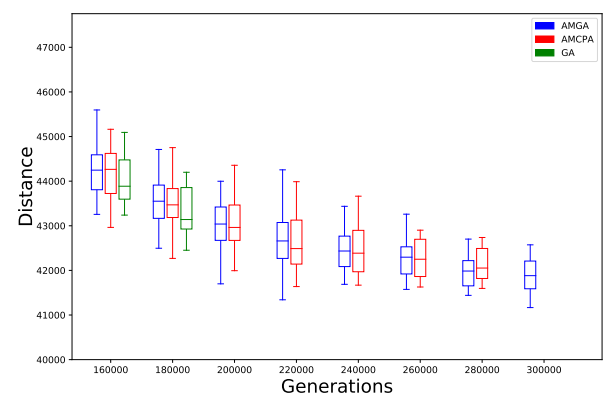

(c) $\mathrm{U} 574(n=574)$

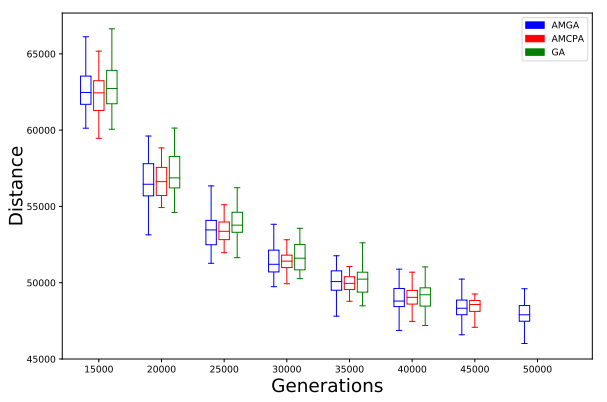

(b) $\operatorname{Lin} 318(n=318)$

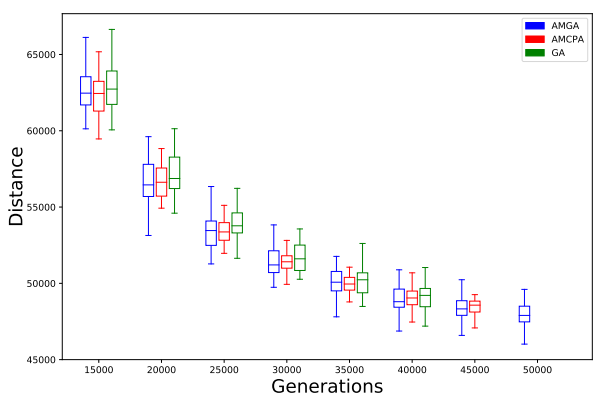

(d) $\operatorname{Rat783}(n=783)$

Fig. 10: Comparison of AMGA, AMCPA, and GA for best of fitness

\section{Conclusion and Future Work}

This paper presents a novel framework called AMGA for an adaptive and modular genetic algorithm. Our framework aims to find the optimal combinations of selection, crossover and mutation operators on a genetic algorithm's outcome and to avoid premature convergence by avoiding genetic bottlenecks. This is done in an on-line manner where different operators are used at different stages of the GA's life. Crossover and mutation operators are selected according to their performance in the previous generations. The selection operator is changed when the current selective pressure does not improve the population's fitness. Experiments demonstrate that AMGA outperforms the existing approaches for solving TSP problem and is tolerant to the complexity and spaces of the instances of TSP. The future work includes the investigation of the effects of adaptive parameter controls on adaptive operator selections.

\section{References}

1. Oliver, I., Smith, D., Holland, J.R.: Study of permutation crossover operators on the traveling salesman problem. In: ICGA (1987) 
2. Murata, T., Ishibuchi, H.: Performance evaluation of genetic algorithms for flowshop scheduling problems. In: CEC, pp. 812-817 (1994)

3. Abdoun, O., Abouchabaka, J., Tajani, C.: Analyzing the performance of mutation operators to solve the travelling salesman problem. International Journal of Emerging Sciences 2, 61-77 (2012)

4. Abdoun, O., Abouchabaka, J.: A comparative study of adaptive crossover operators for genetic algorithms to resolve the traveling salesman problem. International Journal of Computer Applications 31(11), 49-57 (2011)

5. Razali, N.M., Geraghty, J., et al.: Genetic algorithm performance with different selection strategies in solving tsp. In: WCE, vol. 2, pp. 1134-1139 (2011)

6. Goldberg, D.E., Deb, K.: A comparative analysis of selection schemes used in genetic algorithms. In: Foundations of genetic algorithms, vol. 1, pp. 69-93 (1991)

7. Črepinšek, M., Liu, S.H., Mernik, M.: Exploration and exploitation in evolutionary algorithms: A survey. ACM Computing Surveys (CSUR) 45(3), 35 (2013)

8. Karafotias, G., Hoogendoorn, M., Eiben, Á.E.: Parameter control in evolutionary algorithms: Trends and challenges. CEC 19(2), 167-187 (2015)

9. Spears, W.M.: Adapting crossover in evolutionary algorithms. In: Evolutionary programming, pp. 367-384 (1995)

10. Riff, M.C., Bonnaire, X.: Inheriting parents operators: A new dynamic strategy for improving evolutionary algorithms. In: International Symposium on Methodologies for Intelligent Systems, pp. 333-341. Springer (2002)

11. Gomez, J.: Self adaptation of operator rates in evolutionary algorithms. In: Genetic and Evolutionary Computation Conference, pp. 1162-1173. Springer (2004)

12. Cruz-Salinas, A.F., Perdomo, J.G.: Self-adaptation of genetic operators through genetic programming techniques. In: GECCO, pp. 913-920. ACM (2017)

13. Montero, E., Riff, M.C.: Self-calibrating strategies for evolutionary approaches that solve constrained combinatorial problems. In: International Symposium on Methodologies for Intelligent Systems, pp. 262-267. Springer (2008)

14. Montero, E., Riff, M.C.: On-the-fly calibrating strategies for evolutionary algorithms. Information Sciences 181(3), 552-566 (2011)

15. Osaba, E., Diaz, F., Onieva, E., Carballedo, R., Perallos, A.: Amcpa: A population metaheuristic with adaptive crossover probability and multi-crossover mechanism for solving combinatorial optimization problems. International Journal of Artificial Intelligence 12(2), 1-23 (2014)

16. Osaba, E., Onieva, E., Carballedo, R., Diaz, F., Perallos, A.: An adaptive multicrossover population algorithm for solving routing problems. In: Nature Inspired Cooperative Strategies for Optimization, pp. 113-124 (2014)

17. Holland, J.H.: Adaptation in natural and artificial systems: an introductory analysis with applications to biology, control, and artificial intelligence. MIT press (1992)

18. Whitley, L.D., et al.: The genitor algorithm and selection pressure: Why rankbased allocation of reproductive trials is best. In: Proc. of the 3rd Intl. Conf. on Genetic Algorithms, vol. 89, pp. 116-123 (1989)

19. Baker, J.E.: Reducing bias and inefficiency in the selection algorithm. In: Proc. of the 2nd Intl. Conf. on Genetic Algorithms and their application, pp. 14-21 (1987)

20. Davis, L.: Applying adaptive algorithms to epistatic domains. In: International Joint Conference on Artificial Intelligence, vol. 85, pp. 162-164 (1985)

21. Whitley, D., Starkweather, T., Shaner, D.: The traveling salesman and sequence scheduling: Quality solutions using genetic edge recombination (1991) 